\section{Wurmeier-Therapie hat Wirkung, bessert aber die Symptome nicht}

\author{
Wurminfektionen können die allergische Reaktionsbereitschaft \\ beeinflussen, das Atopierisiko reduzieren und den Verlauf von
}

Asthma bronchiale günstig beeinflussen. Ob sich die von den Darmparasiten ausgelösten immunologischen Veränderungen auch für

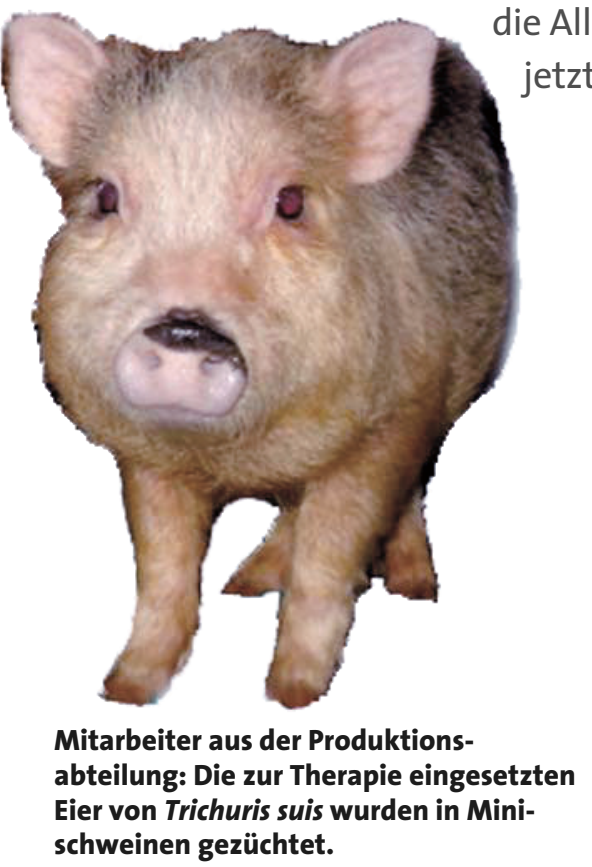

$\mathrm{N}$ achdem bereits Patienten mit entzündlichen Darmerkrankungen erfolgreich mit den T-Zell-modulierend wirkenden Eiern von Trichuris suis (Schweine-Peitschenwurm) behandelt werden konnten, erfolgte nun ein doppelblinder und plazebokontrollierterTherapieversuch bei 100 erwachsenen Patienten mit einer Pollenrhinitis. Eingesetzt wurden dabei in Minischweinen gezüchtete Eier von T. suis, die den Patienten in wässriger Lösung mit jeweils 2.500 entwicklungsfähigen Eiern pro Einzeldosis in dunkel eingefärbten Trinkampullen bereitgestellt wurden. Die Plazebolösung war geruchlich und farblich nicht von Verum zu unterscheiden. Die Behandlung dauerte 21 Tage.

\section{Leukotrienantagonist sorgt für mehr Lebensqualität}

\author{
Patienten mit einer allergischen Rhinitis erleiden deutliche Einbußen \\ ihrer Lebensqualität: Der Schlaf ist gestört, die Konzentrations- und \\ Leistungsfähigkeit herabgesetzt. Ein antiallergisches Medikament \\ sollte die Lebensqualität wieder erhöhen.
}

$\mathrm{D}$ ie Beeinflussung der krankheitsbezogenen Lebensqualität war das primäre Studienziel einer monozentrischen, randomisierten und plazebokontrollierten Doppelblindstudie mit 46 Patienten mit einer seit mindestens zwei Jahren bestehenden persistierenden allergischen Rhinitis. 24 Patienten erhielten einen Monat lang oral $10 \mathrm{mg}$ Montelukast einmal täglich, 24 Patienten entsprechend Plazebo. Vor und nach der Therapie wurden die Patienten anhand des Rhinoconjunctivitis Quality of Life Questionnaire (RQLQ) befragt. Der Fragebogen enthält sieben Domänen mit insgesamt 28 Kritierien wie zum Beispiel Schlaf, nichtallergische Symptome, praktische Probleme und Aktivitäten des täglichen Lebens. Gemessen wurden Veränderungen im RQLQ im Vergleich zu den Ausgangswerten innerhalb einer Gruppe.

In der Montelukast-Gruppe ergab sich ein größerer Rückgang des GesamtRQLQ-Scores im Vergleich zur Plazebogruppe $(\mathrm{p}<0,001)$. Die Unterschiede
Patienten unter Verum $(\mathrm{n}=49)$ entwickelten im Vergleich zu Patienten unter Plazebo $(n=47)$ deutlich häufiger eine vorübergehende Diarrhoe (33\% vs. $2 \%)$ und zeigten erhöhte Eosinophilenzahlen ( $p<0,001)$ sowie erhöhte SerumTiter von T.-suis-spezifischem $\operatorname{IgE}(\mathrm{p}<$ 0,05), $\operatorname{IgG}(\mathrm{p}<0,001), \operatorname{IgG} 4(\mathrm{p}<0,003)$ und $\operatorname{IgA}(\mathrm{p}<0,001)$. Bei den primären Studienzielen, der Veränderung der durchschnittlichen Rhinokonjunktivitissymptome sowie der weitgehend symptomfreien Tage während der Pollensaison im Vergleich zum Vorjahr, ergaben sich dagegen keine statistisch signifikanten Unterschiede zwischen den beiden Behandlungsarmen.

Fazit: Eine 21-tägige Behandlung mit lebensfähigen Eiern des Schweine-Peitschenwurms induziert zwar deutliche infektionstypische Immunantworten, zeigt jedoch keinen Einfluss auf die klinische Symptomatik von Patienten mit einer Pollenrhinitis.

Bager $\mathbf{P}$ et al. Trichuris suis ova therapy for allergic rhinitis: A randomized, doubleblind, placebo-controlled clinical trial. J Allergy Clin Immunol 2010; 125: 123-30

bei den Scores zu Behandlungsbeginn und am Ende des ersten Monats bei allen Einzeldomänen waren sowohl in der Plazebo- als auch der Verumgruppe statistisch signifikant. Bei den Einzeldomänen Schlaf, praktische Probleme, nasale Probleme sowie Aktivitäten, die durch die Augen- und Nasensymptome beeinträchtigt wurden, waren die Gruppendifferenzen jeweils statistisch signifikant zugunsten der Verumbehandlung. Die Verträglichkeit von Montelukast war gut.

Fazit: Die Gabe von Montelukast verbessert die krankheitsspezifische Lebensqualität von Patienten mit einer perennialen allergischen Rhinitis.

$b k$

Cingi C et al. Effects of montelukast on quality of life in patients with persistent allergic rhinitis. Otolaryngol Head Neck Surg 2010; 142: 654-8 